\title{
Lacas y Selladores para Madera a partir de Resinas Alquídicas Obtenidas de Aceites de Higuerilla, Palma y Usados de Fritura
}

\author{
David Ocampo, Elkin D. Aguirre, Alexander Osorio y Luis A. Rios \\ Universidad de Antioquia UdeA, Grupo Procesos Químicos Industriales, Facultad de Ingeniería, Calle 70 \\ No.52-21, Medellín-Colombia. (e-mail: lariospfa@gmail.com;davidocampoe@gmail.com \\ alexosorio979@gmail.com;;elkaiva73@gmail.com)
}

Recibido Ago. 20, 2013; Aceptado Oct. 9, 2013; Versión final recibida Nov. 5, 2013

\begin{abstract}
Resumen
Se presentan resultados de la elaboración de lacas y selladores para madera a partir de resinas alquídicas empleando aceites autóctonos crudos de higuerilla, palma y de fritura para reemplazar aceites importados como el de soja. Los productos desarrollados fueron caracterizados mediante criterios de desempeño tales como brillo, viscosidad, adherencia al sustrato, estabilidad térmica y tiempos de secado, bajo normas nacionales e internacionales, con el fin de determinar similitud o cambios con respecto a contratipos comerciales. El desarrollo plantea la inclusión de este tipo de materias primas, autóctonas de la región, como una alternativa para reducción de costos, generación de empleos directos e indirectos y la incorporación de estos aceites vegetales de bajo valor comercial en la cadena productiva de recubrimientos para madera. Además se da valor agregado a los aceites recuperados de fritura convirtiéndolos en una materia prima de interés comercial y minimizando su impacto ambiental en cuerpos de agua.
\end{abstract}

Palabras clave: resinas alquídicas, aceite de palma, aceite de higuerilla, aceite de fritura recuperado, lacas, selladores, maderas

\section{Wood Coatings and Sealants from Alkyd Resins Obtained from Castor Oil, Palm Oil and used Frying Oil}

\begin{abstract}
Results on the development of new coatings and sealants for wood based on alkyd resins are presented. Indigenous castor and palm oils as well as used cooking oils were used to replace imported soybean oil in the synthesis of the resins. The products developed were characterized in terms of brightness, viscosity, adhesion to the substrate, thermal stability and drying times, under international and national standards, in order to determine similarity or changes with respect to commercial dupes. Is results indicate the possible inclusion of such raw materials, indigenous to the region, as an alternative for reducing costs, generating direct and indirect jobs and the incorporation of these vegetable oils of low commercial value in the production chain of wood coatings. Besides, added value is given to recovered frying oils by making them raw materials of commercial interest and minimizing their environmental impact on water ecosystems.
\end{abstract}

Keywords: alkyd resins, palm oil, castor oil, used frying oil, coatings, sealants, wood 


\section{INTRODUCCIÓN}

Dentro de los materiales de construcción de puertas, ventanas, pisos, baños, cocinas y mobiliario de oficina la madera, que es uno de los materiales más nobles que ofrece la naturaleza, continúa siendo el material más versátil empleado por el hombre con una producción cercana a 4.100 millones de metros cúbicos por año, de los cuales el $56 \%$ es utilizado como combustible, fundamentalmente en países subdesarrollados, los cuales poseen la mayoría de las reservas boscosas de especies latifoliadas, y el $44 \%$ restante es industrializado, predominando la utilización de maderas de coníferas para las diferentes aplicaciones. La importancia de la madera radica en su relativo bajo costo frente a materiales metálicos o plásticos, y que es un producto de fácil manejo, de buena resistencia mecánica, térmica y acústica, además de generar acabados con gran belleza natural. La durabilidad de los productos terminados de madera depende en gran medida del tipo de tratamiento superficial a que es sometido (Garay 2009), a fin de proteger la superficie de agentes externos químicos o biológicos, y esto en gran medida depende del tipo de recubrimiento que se aplica ya sea para inmunizarla, impermeabilizarla o darle un acabado especial. La exposición a toda clase de agentes externos, tales como la humedad y la sequedad procedente de los rayos del sol, puede dañar en gran medida su belleza y calidad, así, los rayos ultravioleta del sol son un enemigo natural de la madera y son los responsables de que ésta, si se encuentra al aire libre, pierda su aspecto natural y adquiera un tono grisáceo. Ello se debe a que el sol degrada la lignina del material. A partir de ahí, la lluvia elimina la lignina, agrietando la madera y abriendo paso a la acción de la humedad. Por su parte, el agua y la humedad no atacan directamente a la madera, pero favorecen las condiciones para el desarrollo de los hongos y los insectos.

Actualmente existen en el mercado una gran diversidad de productos empleados como recubrimientos para madera, que son usados comúnmente para dar tratamiento de primers (selladores de poros) o para el acabado decorativo a las superficies (lacas brillantes). Entre los productos comerciales más usados se destacan los recubrimientos basados en resinas nitrocelulósicas, epóxicas, poliuretánicas, vinílicas, acrílicas, poliésteres y alquídicas (Franco, et al 2009; Marrion, et al 2004; Cardeño, et al 2013). En el caso de las resinas alquídicas, estas últimas son materiales poliméricos derivados de la reacción de polioles y poliácidos, que son modificados con aceites y ácidos grasos naturales insaturados o sintéticos que se caracterizan por su secado oxidativo a temperatura ambiente en presencia de catalizadores y brindar propiedades mecánicas a la película formada (Panda, 2010, Uzoh, et al 2013). En general, en la elaboración de recubrimientos, las resinas alquídicas se mezclan con frecuencia con otros productos tales como las amino-resinas eterificadas (resinas derivadas de la reacción entre el formol, el isobutanol y la úrea) o son modificadas con resinas poliuretánicas, poliamidas, siliconas, monómeros acrílicos y estirénicos o reducibles en agua por emulsificación para mejorar su velocidad de curado, la dureza y el aspecto, generándose productos de bajo costo, fácil aplicación y buen desempeño (Patton, 1962).

En el presente trabajo, se evalúa el uso de diferentes aceites crudos y usados como sustitutos del aceite de soja en la elaboración de las resinas alquídicas y su efecto en las propiedades finales de la misma resina (Pramanik et al., 2012), así como las propiedades finales de los primers (selladores) y lacas brillantes para madera en que fueron utilizadas. Se evaluaron los aceites saturados de higuerilla, palma y el aceite de fritura recuperado para la obtención de resinas alquídicas que cumplan con los estándares de calidad para Colombia para la obtención de productos de alto interés comercial como los son los selladores y las lacas catalizados para madera según los criterios de calidad de la Norma Técnica Colombiana (NTC 1651).

\section{PROCEDIMIENTO EXPERIMENTAL}

\section{Materiales y Equipos}

En la elaboración de las resinas alquídicas se emplearon aceites vegetales crudos de palma e higuerilla obtenidos de productores locales, aceite usado de fritura recolectado de restaurantes hospitalarios (Hospital San Vicente de Paul) y aceite de soja RBD obtenido del grupo comercializador TEAM. Como fuente de polioles se emplearon pentaeritritol (BellChem Internacional), Monotilenglicol (Merck) yglicerina comercial grado USP (Merck). Como fuente de poliácidos se utilizó anhídrido ftálico grado analítico (Panreac) y anhídrido maléico grado analítico (Panreac). Se emplearon como catalizadores el acetato y el óxido de plomo (Merck), ricinoleato de litio al 50\% (Aldar Química) e hidróxido de sodio en escamas (Merck). En la elaboración de las lacas y selladores catalizados al ácido se emplearon como solventes xilol, isobutanol, Butilcellosolve y etanol grado comercial, resina úrea-formol isobutilada, de un productor local, resina melaminaúrea formol isobutilada, de un productor local, talco blanco malla 400 (Alfaquimicos LTDA.), caolín blanco malla 400 (Exdequin S.A.), pentonita grado comercial, dispersante Claytone 40 (Rockwood additives), aditivo de nivelación Lisoperse (Conquimica S.A.). Las pruebas de desempeño reportadas en el presente artículo se llevaron a cabo mediante el uso de equipos tales como: medidor de brillo (BYK MicroTri-gloss 1088865) bajo norma ASTM D523, horno de convección forzada (Memmert) para determinación y 
ajuste del contenido de sólidos, viscosímetro copa Ford N4 (BYK) bajo norma ASTM D1200-10, Aplicador de película (BYK).

\section{Adecuación y caracterización de aceites crudos y usados}

El aceite crudo de higuerilla obtenido por extracción de semillas de la variedad VC/06-99, conocida como Negra Jaspeada fue sometido a un proceso de desgomado a $80{ }^{\circ} \mathrm{C}$ con agua acidulada con $\mathrm{H}_{3} \mathrm{PO}_{4}$, con una etapa posterior de neutralización con solución alcalina de $\mathrm{NaOH}$, seguida de centrifugación a 8000 rpm por $30 \mathrm{~min}$, lavado con agua y posterior secado por roto evaporación (Sevim, et al 2005). El aceite de palma crudo fue sometido a un proceso de neutralización de la acidez libre empleando solución alcalina de $\mathrm{NaOH}$, el aceite usado de fritura fue sometido a un proceso de desecación con agitación constante empleando $\mathrm{NaCl}$ y $\mathrm{Na}_{2} \mathrm{SO}_{4}$ a una temperatura entre $75-80^{\circ} \mathrm{C}$ durante una hora con posterior decoloración y retención de impurezas usando carbón activado como material adsorbente, decantación, filtración de las impurezas sólidas y neutralización de la acidez remante (Cyengros, et al 2004; Kulkarni, et al 2006; Zahira, et al 2013). Los productos obtenidos fueron caracterizados (tabla 1) para determinar las condiciones fisicoquímicas finales según normas ASTM para índice de yodo, saponificación, acidez, hidroxilo, humedad (ASTM D 5554; ASTM D 5558-95; ASTM D 1980-87; ASTM D 1957-86) y la norma NTC 4967 para determinar la composición química.

Tabla 1: Caracterización de aceites vegetales (n.a: no aplica)

\begin{tabular}{lcccc}
\hline Índice Medido & Aceite de fritura & Palma & Higuerilla & Soja \\
\hline Yodo (g l2/100 g muestra) & 100,9 & 56,5 & 88,5 & 139,1 \\
$\begin{array}{l}\text { Saponificación (mg } \\
\text { muestra) }\end{array}$ & 190,7 & 198,3 & 181,5 & 193,3 \\
Acidez(mg KOH/g muestra) & 1,7 & 0,35 & 0,53 & 1,02 \\
Hidroxilo(mg KOH/g muestra) & n.a & n.a & 162,5 & n.a \\
Humedad (\% peso) & 0,08 & 0,12 & 0,06 & 0,15 \\
\hline
\end{tabular}

Fabricación de las resinas alquídicas vía glicerólisis de los aceites crudos y usados

La tabla 2 muestra el porcentaje utilizado de cada materia prima en la fabricación de las resinas alquídicas. Estas resinas fueron preparadas en dos etapas: La primera etapa (Fase I) correspondiente a la glicerólisis de los aceites empleando como catalizador $\mathrm{NaOH}$ (Patton, 1962; Cardona, et al 2010). En ésta etapa se llevó a cabo la alcohólisis individual de cada aceite vegetal (palma, higuerilla, fritura, soja RBD importado).

Tabla 2: Formulación típica de una resina alquídica corta en aceite (n.a: no aplica)

\begin{tabular}{lcc}
\hline Materia Prima & \% peso & Funcionalidad química \\
\hline Aceite o ácido graso & & \\
Aceite de soja & 20 & 1 \\
Aceite de higuerilla & 20 & 2 \\
Aceite de Palma & 20 & 1 \\
Aceite de Fritura & 20 & 1 \\
Recuperado & & \\
Fuente de polioles & 5 & 2 \\
Glicerina & 10 & 4 \\
Pentaeritritol & 5 & 2 \\
Etilenglicol & & \\
Fuente de poliácidos & 19 & 2 \\
Anhídrido ftálico & 0,1 & 2 \\
Anhídrido maléico & & \\
Solvente de dilución & 40,9 & n.a \\
Xilol & & \\
\hline
\end{tabular}

La segunda fase del proceso (Fase II Policondensación) correspondiente a la etapa de esterificación de los monoglicéridos obtenidos, consta de hacer reaccionar dichos compuestos con polioles y poliácidos previamente seleccionados como materias primas de grado comercial (Patton, 1962). La síntesis de las resinas poliméricas es llevada a cabo en reactores de vidrio de $500 \mathrm{ml}$ provistos de sistema de reflujo, 
agitación mecánica y alimentación de nitrógeno como gas inerte, para evitar la oxidación de los productos de reacción. El calentamiento se llevó a cabo empleando mantas de calentamiento con control de temperatura (hasta $230-240{ }^{\circ} \mathrm{C}$ ). En la etapa de esterificación, la reacción fue monitoreada por determinación periódica del valor ácido de la mezcla hasta alcanzar valores menores a $10 \mathrm{mg} \mathrm{KOH} / \mathrm{g}$ (Alireza, et al 2013). El valor ácido de la resina alquídica obtenida se determina usando la norma ASTM D 1639-90. Los detalles de fabricación de este tipo de resinas son descritos en publicaciones anteriores (Cardeño, et al 2013, Uzoh, et al 2013).

Preparación y caracterización de lacas y selladores catalizados al ácido.

Para determinar la calidad de las resinas alquídicas obtenidas se fabricaron productos de interés comercial en la línea de los recubrimientos tales como lacas y selladores catalizados al ácido empleando formulaciones típicas de empresas locales (ver tabla 3.) (Ayca, et al 2010; Lisperguer, et al 2002). Las materias primas se mezclan durante 1 hora empleando agitación con disco Cowles hasta alcanzar una completa homogenización del sistema. Posteriormente los productos obtenidos son aplicados sobre la superficie de una lámina de madera de pino con pistola a presión. El recubrimiento es sometido a diferentes pruebas de caracterización como adherencia al sustrato (Norma NTC 811), percepción de la apariencia de brillo (ASTM D523), tiempos de secado sobre vidrio (ASTM D1640), viscosidad copa Ford \#4 (ASTM D120010), lijabilidad y pruebas de ciclos térmicos. (Ayca, et al 2010).

Tabla 3: Formulaciones típicas para sellador y laca brillante (\% en masa)

\begin{tabular}{lclc}
\hline & Sellador & & \multicolumn{2}{c}{ Laca brillante } \\
\hline Materia prima & $\%$ & Materia Prima & $\%$ \\
Resina alquídica & 26 & Resina Alquídica & 41 \\
Xilol & 34 & Xilol & 15 \\
Aditivo nivelador & 1,5 & Resina comercial úrea-formol- melamina & 1,5 \\
Talco blanco & 4,6 & Resina comercial úrea formaldehido isobutilada & 27 \\
Caolín blanco & 1,8 & Aditivo nivelador & 0,04 \\
Talco industrial malla 400 & 2,2 & Isobutanol & 12 \\
Pentonita & 0,5 & Butilcellosolve & 2,8 \\
Resina comercial úrea formaldehido isobutilada & 18 & & \\
Etanol & 7 & Claytone 40 & 0,66 \\
2 Etil-hexil-acetato & 1,4 & & \\
Isobutanol & 3 & & \\
\hline
\end{tabular}

\section{RESULTADOS Y DISCUSIÓN}

\section{Caracterización de las resinas alquídicas}

Las propiedades fisicoquímicas de las resinas alquídicas para diferentes mezclas de aceites crudos y de fritura con aceite de soja son presentadas en la tabla 4.

Tabla 4: Propiedades fisicoquímicas finales de las resinas alquídicas

\begin{tabular}{cccccc}
\hline Ensayo & $\%$ sólidos & Aceites utilizados & Viscosidad Gardner & Color Gardner & Índice Acidez \\
\hline 1 & 57,01 & Aceite de Higuerilla & Q-R & 1 & $<10$ \\
2 & 58,36 & Aceite de Soja (resina comercial) & Z4-Z5 & 3 & $<10$ \\
3 & 54,09 & Aceite de Palma & W-X & $5-6$ & $<10$ \\
4 & 56,96 & Aceite de Fritura recuperado & Z-Z1 & $7-8$ & $<10$ \\
\hline
\end{tabular}

El rango o valor ajustado y recomendado en cuanto al porcentaje de sólidos para resinas alquídicas es de $57,0 \pm 2,0 \%$. Los valores para resinas alquídicas comerciales en Colombia oscilan en las cantidades que reportan los resultados de la tabla 4 , según los requerimientos de calidad basados en las normas técnicas NTC 1651, NTC 1283, NTC 1401 y NTC 1786.Para recubrimientos alquídicos la viscosidad se establece según requerimientos de NTC 1651 y NTC 996 los cuales buscan garantizar la calidad en la aplicación de recubrimientos tipo alquídicos (nivelación y chorreo), como era de esperarse la viscosidad de las resinas 
obtenidas depende del índice de insaturaciones de los aceites, por lo cual a mayor índice de yodo, mayor es la viscosidad como se puede observar en la tabla 4; sin embargo debido a los grupos hidroxilos en la estructura química del aceite de higuerilla, éste puede formar cadenas más largas de resinas, por lo que existe una menor reticulación y a su vez la viscosidad será más baja.

El parámetro de color presenta un valor recomendado para resinas comerciales no mayor a 3 en escala de color Gardner para aplicaciones en lacas, barnices y esmaltes para acabados, mientras que valores más altos de color se pueden usar en preparadores de superficie como anticorrosivos para metales y selladores para madera. Este punto por lo general es establecido por criterios de calidad de las empresas y se referencia en el numeral 3.4 de la norma NTC 1651. El rango de acidez se estableció de acuerdo a la experiencia propia y a la consulta de otros autores (Khurshid, et al 2006). El índice de acidez se mide con base en la resina solventada (no en base seca) y se recomiendan índices de acidez no mayores a 10.

De acuerdo a la tabla 4 y a los valores reportados en la literatura técnica (Patton, 1962; Khurshid, et al 2006), las resinas sintetizadas cumplen con los parámetros de calidad establecidos en la industria de las resinas alquídicas; se exceptúa el valor de la viscosidad del ensayo 1 que pueden ocasionar problemas de aplicación como chorreo y mala nivelación del recubrimiento debido a su baja viscosidad.

\section{Análisis de desempeño en selladores y lacas para madera}

En la tabla 5 se reportan las propiedades fisicoquímicas y de desempeño para cada uno de los selladores y las lacas utilizadas para la protección y aplicación en madera elaborados a partir de las resinas alquídicas obtenidas con los diferentes aceites. La viscosidad en copa Ford \#4 a $20^{\circ} \mathrm{C}$ (segundos) es un parámetro importante para la determinación de la calidad del sellador y la laca formulados. Viscosidades muy bajas de los recubrimientos generan problemas de nivelación, chorreo, secado y formación de película; así mismo viscosidades muy altas presentan inconvenientes de brochabilidad, homogeneidad del recubrimiento aplicado, taponamiento de dispositivos de aplicación, entre otros. En la tabla 5 también se muestran los resultados de la comparación de las viscosidades en copa Ford \#4 para cada uno de los ensayos realizados.

Se puede observar que para selladores comerciales fabricados con resinas alquídicas de soja, la viscosidad medida para copa Ford $\mathrm{N}^{\circ} 4$ se encuentra entre 10 y 18 segundos y para las lacas entre 30 y 40 segundos. Por lo general la tendencia de los ensayos realizados muestran viscosidades entre los rangos de calidad (22-100 segundos ASTM D1200-10), a excepción de los ensayos realizados con aceites de palma que aumentan significativamente frente a los demás, sin embargo las demás pruebas para estos ensayos no mostraron problemas importantes en la aplicación. Se considera que las resinas alquídicas fabricadas con aceite de palma también cumplen con los criterios de calidad exigidos por los recubrimientos (ASTM D120010) (Lacas y selladores para madera).

Tabla 5: Propiedades de los selladores obtenidos con cada resina alquídica

\begin{tabular}{|c|c|c|c|c|c|c|c|c|c|c|c|}
\hline \multirow{2}{*}{$\begin{array}{l}\text { Ens } \\
\text { ayo }\end{array}$} & \multirow{2}{*}{$\begin{array}{c}\% \\
\text { Sólido } \\
\text { s }\end{array}$} & \multirow{2}{*}{$\begin{array}{l}\text { Resina alquídica } \\
\text { corta en aceite }\end{array}$} & \multirow{2}{*}{$\begin{array}{l}\text { Viscosidad Copa } \\
\text { Ford sellador }\end{array}$} & \multirow{2}{*}{$\begin{array}{l}\text { Viscosidad } \\
\text { Copa Ford } \\
\text { Laca }\end{array}$} & \multirow{2}{*}{$\begin{array}{l}t \text { de secado } \\
\text { sellador (min) }\end{array}$} & \multicolumn{3}{|c|}{$\begin{array}{l}\text { t de secado de } \\
\text { la laca (min) }\end{array}$} & \multicolumn{3}{|c|}{$\begin{array}{l}\text { Brillo de } \\
\text { Lacas (UG) }\end{array}$} \\
\hline & & & & & & $\begin{array}{l}\text { Pol } \\
\text { vo }\end{array}$ & $\begin{array}{l}\mathrm{Ta} \\
\text { cto } \\
\end{array}$ & $\begin{array}{l}\text { Sec } \\
\text { ado }\end{array}$ & $20^{\circ}$ & $60^{\circ}$ & $85^{\circ}$ \\
\hline 1 & 57,01 & Higuerilla & 19 & 32,81 & 9,18 & $\begin{array}{l}13, \\
23\end{array}$ & $\begin{array}{l}29 \\
02\end{array}$ & $\begin{array}{c}40,4 \\
7\end{array}$ & $\begin{array}{c}15 \\
1\end{array}$ & $\begin{array}{c}14 \\
1\end{array}$ & $\begin{array}{c}11 \\
2\end{array}$ \\
\hline 2 & 54,09 & Palma & 27,53 & 69,1 & 45,12 & $\begin{array}{c}6,5 \\
2\end{array}$ & $\begin{array}{l}16 \\
30\end{array}$ & $\begin{array}{c}23,2 \\
2\end{array}$ & $\begin{array}{c}16 \\
7\end{array}$ & $\begin{array}{c}14 \\
8\end{array}$ & $\begin{array}{c}11 \\
0\end{array}$ \\
\hline 3 & 56,96 & Fritura & 17,63 & 31,1 & 16,21 & $\begin{array}{c}7,1 \\
8\end{array}$ & $\begin{array}{l}14 \\
70\end{array}$ & $\begin{array}{c}19,6 \\
7\end{array}$ & $\begin{array}{l}16 \\
6\end{array}$ & $\begin{array}{l}14 \\
7\end{array}$ & $\begin{array}{c}11 \\
0\end{array}$ \\
\hline 4 & 57,40 & $\begin{array}{l}\text { Soja (contratipo } \\
\text { comercial) }\end{array}$ & 10,50 & 39 & 17,00 & $\begin{array}{c}7,6 \\
7\end{array}$ & $\begin{array}{l}14, \\
22\end{array}$ & $\begin{array}{c}27,4 \\
3\end{array}$ & $\begin{array}{c}15 \\
7\end{array}$ & $\begin{array}{c}14 \\
3\end{array}$ & $\begin{array}{c}11 \\
2\end{array}$ \\
\hline
\end{tabular}

La medición de los valores de tiempo de secado se llevaron a cabo siguiendo los lineamientos de la norma ASTM D1640 para recubrimientos. El ensayo 4, soja, representa un sellador estándar fabricado con resina alquídica de contratipo comercial a base de aceite de soja RBD. Dicho sellador debe tener un tiempo de secado menor de 25 minutos a condiciones ambientales $\left(50 \%\right.$ humedad, $\left.23-25^{\circ} \mathrm{C}\right)$, el resultado del análisis del sellador muestra un tiempo de secado alrededor de los 17 minutos. Según los resultados obtenidos se observan ensayos con tiempos de secado por debajo de los 10 minutos, lo cual es un resultado muy interesante pues es un indicio de que las resinas obtenidas presentan buenas propiedades de curado y formación de película por entrecruzamiento (crosslinking) y presentan reacciones de curado por 
rompimiento u oxidación de los dobles enlaces o insaturaciones de la cadena polimérica. En forma general los valores en los tiempos de secado obtenidos no superan, en la mayoría de ensayos, los valores máximos permitidos por el control de calidad de este tipo de productos (para selladores comerciales tiempos de secado $\leq 25$ minutos) a excepción del ensayo realizado con aceite de palma, el cual presenta un tiempo de secado de 45 minutos, debido al bajo índice de yodo, ya que no posee gran cantidad de insaturaciones factor principal para el curado de las resinas de este tipo.

Los tiempos de secado obtenidos según la norma ASTM D1640 para los diferentes ensayos en las lacas catalizadas no difieren mucho de su contratipo comercial, incluso los ensayos con palma y fritura muestran valores por debajo del estándar de soja, mientras que el ensayo con higuerilla, sí muestra unos tiempos de secado mayores al estándar comercial, pero aún siguen siendo resultados interesantes para la generación de los productos comerciales. El ensayo con resina alquídica de higuerilla es el que posee un bajo porcentaje de insaturaciones, por lo cual es posible que la reticulación al aire de esta resina sea mucho más lenta que las de sus contratipos de fritura e incluso el de soja comercial; sin embargo su bajo contenido de insaturaciones le proporciona características de alto interés comercial para recubrimientos de alto desempeño en la fabricación de lacas y esmaltes no amarillentables o no oxidables.

La medición de los índices de brillo se llevó a cabo según los lineamientos de la norma ASTM D523. En esta medición se toma un blanco comparativo (laca comercial estándar fabricada con aceite de soja altamente insaturado), dicho blanco reporta valores para diferentes ángulos:

Brillo en ángulo $20^{\circ}: \quad 157$ UG

Brillo en ángulo de $60^{\circ}: 143 \mathrm{UG}$

Brillo en ángulo de $85^{\circ}: 112$ UG

Para los ensayos realizados con los diferentes aceites como sustitutos de aceite de soja, se puede observar que las propiedades de acabado en cuanto al brillo de los recubrimientos (tipo lacas) obtenidos no se ven afectados por el cambio realizado en la formulación, por tanto cumplen satisfactoriamente con los índices de calidad en brillo para los recubrimientos de acabado (lacas catalizadas al acido para aplicación en maderas) y pueden ser recomendadas ampliamente como desarrollo tecnológico adaptable a nivel industrial siempre y cuando se garantice la disponibilidad y tratamiento de los aceites autóctonos analizados como sustitutos de aceites insaturados de alto costo como el de Soja. Aunque las diferencias en color de las resinas son notorias, la calidad de los productos obtenidos para la generación de lacas y recubrimientos de acabado no muestra cambios realmente importantes en los productos obtenidos, por lo cual todas las aplicaciones cumplen con los límites permisibles de calidad para el parámetro de brillo de los recubrimientos.

Un caso importante se presenta cuando se emplea el aceite de higuerilla al $100 \%$, dando como resultado una resina alquídica de muy buen aspecto con un valor en escala de color Gardner $\leq 1$. Este tipo de resina puede ser empleada para la fabricación y obtención de lacas y esmaltes de alto desempeño como lacas no amarillentables (bajo índice de oxidación y amarillentamiento con el tiempo) o esmaltes no amarillentables. Los selladores presentaron buen lijado para todos los ensayos debido a que se obtuvo un secado final menor a una hora, la lija no se adhería ni se presentaba tactosidad lo cual conlleva a desprendimiento de cargas junto con la resina polimérica que compone los selladores.

\section{CONCLUSIONES}

A partir de los resultados obtenidos se puede concluir que la calidad de los recubrimientos, la cual depende directamente de la calidad de las resinas poliméricas utilizados (Alireza, et al 2013), cumple con los criterios definidos para los productos (Recubrimientos) como tipos 1 y 2 según la norma técnica NTC1651, sin llevar a cabo modificaciones químicas ni adición de agentes reticulantes o secantes.

La comparación técnica de los recubrimientos fabricados con resinas autóctonas (de bajo contenido de insaturación) frente a productos comerciales obtenidos a partir de aceite de soja importado (altamente insaturado), no muestra cambios muy marcados en cuanto a la calidad de los mismos, por lo cual dichos ensayos (obtención de lacas y selladores para madera a partir de resinas alquídicas formuladas con aceites crudos y usados recuperados) muestran viabilidad e interés comercial para los productores locales, puesto que se cumple con los criterios de calidad asociados a las normas técnicas Colombianas (NTC 1651) y se propende por reemplazar algunas materias primas importadas por autóctonas, lo que impulsa la cadena productiva de este tipo de especies en Colombia (higuerilla y palma) generando empleos directos e indirectos, así como una reducción en los costos de los fletes e impuestos pagados por la importación de aceites insaturados de alto costo. 
El uso de aceites de fritura usados y recuperados es una estrategia técnica y ecológicamente viable para la obtención de resinas alquídicas y su aplicación en procesos productivos puesto que genera valor agregado al residuo, el cual es generalmente vertido a los cuerpos de agua tanto en zonas urbanas como rurales de nuestro país y del mundo en general, ayudando así a cerrar el ciclo productivo de los aceites comestibles.

\section{AGRADECIMIENTOS}

El desarrollo de este trabajo fue financiado gracias al apoyo de COLCIENCIAS, a través del Patrimonio Autónomo Fondo Nacional de Financiamiento para la Ciencia, la Tecnología y la Innovación, Francisco José de Caldas, de la UNIVERSIDAD DE ANTIOQUIA yFundación para la Promoción de la Investigación y la Tecnología del Banco de la Republica

\section{REFERENCIAS}

Alireza A.N., Rosiyah, Y., Seng-Neon, G, Cleaner production through using by-product palm stearin tosynthesis alkyd resin for coating applications. Journal of Cleaner Production: 54 307-314 (2013)

ASTM D523-08: Standard Test Method for Specular Gloss", Annual Book of ASTM Standards, United States (2008).

ASTM D1200-10: Standard Test Method for Viscosity by Ford Viscosity Cup", Annual Book of ASTM Standards. United States (2010).

ASTM D1639-90: Standard Test Method for Acid Value of Organic Coating Materials. West Conshohocken, PA, United States (1990).

ASTM D1640: Standard Test Methods for Drying, Curing, or Film Formation of Organic Coatings at Room Temperature, Annual Book of ASTM Standards, United States (2009).

ASTM D1957-86: Standard Test Method for Hydroxyl Value of Fatty Oils and Acids. West Conshohocken, PA, United States (2001).

ASTM D1980-87: Standard Test Method for Acid Value of Fatty Acids and Polymerized Fatty Acids. West Conshohocken, PA, United States (1998).

ASTM D5554: Standard test method for determination of the iodine value of fats and oils. West Conshohocken, PA, United States (2011).

ASTM D5558-95: Standard Test Method for Determination of the Saponification Value of Fats and Oils. West Conshohocken, PA, United States (2011).

Ayca, B., Gamze, G., Isıl, A. y Tülin B.I, Effects of urea formaldehyde resin to film properties of alkydmelamine formaldehyde resins containing organo clay, Progress in Organic Coatings: 68 363-365 (2010)

Cardeño, F.; Rios, L; Cardona, F; Ocampo, D. Síntesis de Resinas Alquídicas a partir de aceites de higuerilla, palma y de fritura mezclados con aceite de soja, Inf. Tecnol. 24(4), en prensa(2013).

Cardona, S.M. y otros cuatro autores, Production of monoglyceridesfrom castor oilusingcrude and refinedglycerin: Study of themain variables of theprocess, Alimentos: Ciencia, Tecnología E Ingeniería, ISSN 0121-4004 (2010-en línea).

Cyengros, J; Cvengrosova, Z. Used Frying oils and Fats and their utilization in the production of methyl esters of higher fatty acids. Biomass Bioenergy 27: 173-81 (2004).

Franco, B. \& Jon, G. "Industrial Wood Coatings: Theory and Practice”, Elsevier, Oxford (2009).

Panda, H., Alkyd Resins Technology Handbook. Asia Pacific Business Press Inc, 1, New Delhi, India (2010).

Garay, Rose Marie. Efectos de dos Protectores Superficiales en las Propiedades de Tableros de Madera después de un Año de Exposición a la Intemperie.Inf. tecnol. 2009, vol.20, n.4, pp. 123-130.

Khurshid, M., Production of alkyd resin. Dhahran: Summer training report, King Fadh University of petroleum and minerals. (2006). 
Kulkarni MG, Dalai AK. Waste Cooking Oils, an economical source for biodiesel: A review.-Ind. Eng. Chem Res; 45:2901-13 (2006).

Lisperguer, J y Droguett, C. Caracterización del curado de resinas urea formaldehido por calorimetría diferencial de barrido (DSC).Bol. Soc. Chil. Quím. 2002, vol.47, n.1, pp. 33-38.

Marrion A., Film formation. The Chemistry and Physics of Coatings, Segunda edición, Published by The Royal Society of Chemistry, 49-59, Northampton, UK, (2004).

NTC811: Método de ensayo para medir la adhesión de un recubrimiento mediante el ensayo de cinta, ICONTEC Bogotá D.C. Colombia (1997).

NTC 996: Pinturas y productos afines. Aplicación en láminas de ensayo, ICONTEC Bogotá DC, Colombia (1975).

NTC1283: Esmaltes Sintéticos de secamiento al aire, ICONTEC Bogotá D.C. Colombia (2001).

NTC1401: Pinturas y productos afines. Barnices alquídicos de secamiento al aire, ICONTEC Bogotá D.C (1986).

NTC 1651: Pinturas. Imprimantes anticorrosivos con vehículo alquídico, ICONTEC Bogotá DC, Colombia (1997).

NTC1786: Pinturas. Determinación del volumen de material no volátil en recubrimientos claros o pigmentados, ICONTEC Bogotá D.C. (2003).

NTC4967: Grasas y Aceites animales y vegetales. Preparación de esteres metílicos de ácidos grasos, ICONTECBogotá D.C. Colombia (2001).

Patton, T.C., Alkyd resin technology, formulating techniques and allied calculations. Manual \# 8. New York: Interscience: 197, (1962).

Pramanik, S., Sagak, K., Konwar, B.K., Karak,.Synthesis, characterization and properties of a castor oil modified biodegradable poly(ester amide) resin. Prog.Org. Coat. 75 (4), 569-578. (2012)

Sevim, Z.E., L. Zengshe y X. Jingyuan, Preparation characterization and mechanical properties of epoxidized soybean oil/clay nanocomposites, Polymer: 46(23), 10119-10127, (2005).

Uzoh, C.F., Onukwuli, O.D., Odera, R.S. y Ofochebe, S., Optimization of polyesterification process for production of palm oil modified alkyd resin using response surface methodology, Journal of Environmental Chemical Engineering: Article in press (2013).

Zahira Y., Masita M., Alherbawi, M. Overview of the production of biodiesel from waste cooking oil. Renewable and Sustainable Energy Reviews 184-193 (2013). 